\title{
Nox4-Mediated Cell Signaling Regulates Differentiation and Survival of Neural Crest Stem Cells
}

\author{
Ji-Eun Lee, Kyu Eun Cho, Kyung Eun Lee, Jaesang Kim*, and Yun Soo Bae*
}

The function of reactive oxygen species (ROS) as second messengers in cell differentiation has been demonstrated only for a limited number of cell types. Here, we used a wellestablished protocol for BMP2-induced neuronal differentiation of neural crest stem cells (NCSCs) to examine the function of BMP2-induced ROS during the process. We first show that BMP2 indeed induces ROS generation in NCSCs and that blocking ROS generation by pretreatment of cells with diphenyleneiodonium (DPI) as NADPH oxidase (Nox) inhibitor inhibits neuronal differentiation. Among the ROSgenerating Nox isozymes, only Nox4 was expressed at a detectable level in NCSCs. Nox4 appears to be critical for survival of NCSCs at least in vitro as down-regulation by RNA interference led to apoptotic response from NCSCs. Interestingly, development of neural crest-derived peripheral neural structures in Nox4-/- mouse appears to be grossly normal, although Nox4-/- embryos were born at a sub-Mendelian ratio and showed delayed over-all development. Specifically, cranial and dorsal root ganglia, derived from NCSCs, were clearly present in Nox4-/- embryo at embryonic days (E) 9.5 and 10.5. These results suggest that Nox4-mediated ROS generation likely plays important role in fate determination and differentiation of NCSCs, but other Nox isozymes play redundant function during embryogenesis.

\section{INTRODUCTION}

Reactive oxygen species (ROS) are generated in response to a diverse array of extracellular stimuli and induce various cellular responses including proliferation, inflammation, and apoptosis (Bae et al., 2011). In particular, the identification and functional analyses of cell-surface receptor-regulated enzyme complexes responsible for ROS production firmly established the role of ROS as second messengers in cell signaling (Bae et al., 2011). The enzymes responsible for synthesis of ROS are members

Department of Life Science and GT5 Program, Ewha Womans University, Seoul 120-750, Korea

*Correspondence: jkim1964@ewha.ac.kr (JK); baeys@ewha.ac.kr (YSB)

Received 4 September, 2014; accepted 23 September, 2014; published online 10 November, 2014

Keywords: bone morphogenetic protein, neural crest stem cell, neuronal differentiation, Nox4, reactive oxygen species of NADPH (nicotinamide adenine dinucleotide phosphate) oxidases (Nox) (Bae et al., 2011). Since the isolation of gp91phox/ NADPH oxidase 2 (Nox2) in phagocytic cells, six other members of Nox family have been discovered to date in human (Bae et al., 2011). Members of this gene family are expressed in a wide variety of cell types either exclusively or in combination, and functional association with growth factor receptors, Gprotein coupled receptors, and Toll-like receptors has been confirmed in numerous cell types (Bae et al., 2011).

Neural crest cells originate along the border of neural plate and epidermal ectoderm during early embryogenesis and give rise to multiple progeny cell types after migration throughout the body (Le Douarin, 1980). Among the cell types produced are neurons and glia of peripheral ganglia, Schwann cells in association with axonal fibers outside the central nervous system, and melanocytes in the inner-most layer of epidermis (Anderson, 1997). For some of these cells, extrinsic factors that induce differentiation from multipotent stem cells are known (Anderson, 1997). This was possible in large part due to the availability of pure clonal primary cell culture system in vitro which enabled definitive cell fate determination. Most notably, Shah and coworkers demonstrated that BMP2 can promote sympathetic neuronal differentiation form rat NCSCs using a clonal culture system (Shah et al., 1996).

Several recent studies have reported that Nox-mediated ROS generation is involved in differentiation of various cell types. For example, redox potential level has been implicated in differentiation of stem cells to cardiac myocytes (Buggisch et al., 2007; Milosevic et al., 2010). Also, activation of Nox4 has been shown to be critical for differentiation of neonatal c-kit ${ }^{+}$cardiac precursor cells to smooth muscle cells (Nadworny et al., 2013). Interestingly, a recent study by Mandal and coworkers showed that ROS generated by Nox4 is essential for BMP2-induced osteoblast differentiation from murine 2T3 pre-osteoblast cells (Mandal et al., 2011). Profibrotic cell differentiation from adult renal progenitor cells through Nox4 activation is also initiated by BMP2 (Simone et al., 2012). Based on such previous reports, we hypothesized that generation of BMP2-inudced ROS in NCSCs is necessary for neuronal differentiation. Here, we demonstrate that ROS is indeed generated in NCSCs via Nox4 activation in response to BMP2 and that ROS is required for efficient neuronal differentiation of NCSCs.

\section{MATERIALS AND METHODS}

Cell culture

NCSCs were prepared from rat embryos 10.5 days post coitus 


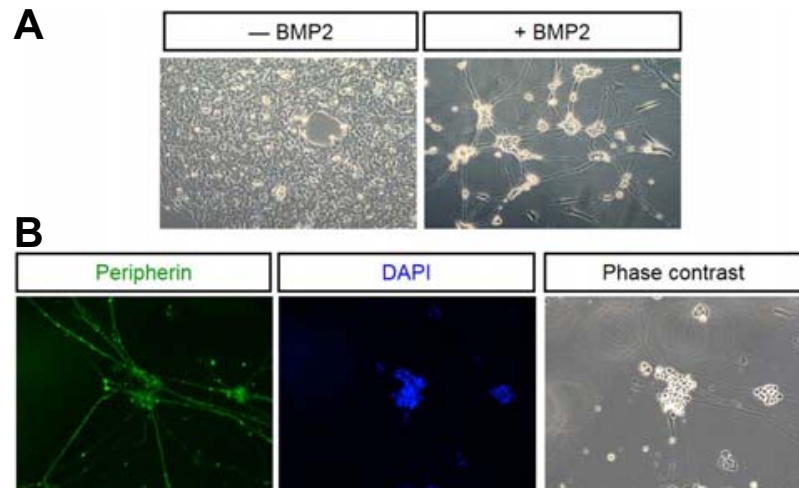

Fig. 1. Neuronal differentiation of NCSCs. (A) Cultures in the absence (left panel) and presence (right panel) of BMP2. Induction of phase bright cells with neurites are seen only with BMP2. (B) BMP2-treated cells are positive for Peripherin, a mature neuronal marker (left panel). Nuclei stained by DAPI (4' 6-Diamidino-2-Phenylindole; middle panel) are seen centrally from which Peripherin positive neurites are extended (left and right panels).

as previously described with minor modifications (Stemple and Anderson, 1992) and cultured in the simplified medium for NCSCs described by Morrison et al. (1999). Recombinant BMP2 was purchased from $R \& D$ Systems and applied to the culture medium at the concentration of $1.6 \mathrm{nM}$ for neuronal differentiation of NCSCs.

\section{Immunocytochemistry and immunoblotting}

The mouse monoclonal anti-Sox10 antibody has been described (Lo et al., 2002). Anti-Nox4 antibody and anti-Peripherin antibody were purchased from Santa Cruz and Millipore respectively. NCSCs were fixed in $4 \%$ paraformaldehyde in PBS and permeabilized with $0.2 \%$ Triton X-100 in PBS supplemented with $10 \%$ goat serum prior to immunostaining. Alexa Red or Alexa Green conjugated goat antibodies against mouse and rabbit antibodies were obtained from Molecular Probes. Anti-Caspase 3 antibody and anti-actin antibody used in immunoblotting assay were respectively obtained from Cell Signaling and Sigma.

\section{RTPCR}

Total RNA was extracted from mouse adipose-derived mesenchymal cells using Qiagen RNA extraction kit (Qiagen). RNA was reverse-transcribed using SuperScript First-Strand Synthesis System for RTPCR kit (Invitrogen), and PCR was performed to determine the expression levels of Nox enzymes. Genes and oligonucleotide primer sequences are provided (Supplementary Table S1). For real time RTPCR, the mRNA levels were measured in duplicates with predesigned TaqMan ${ }^{\circledR}$ primer and probe sets purchased from Applied Biosystems and normalized by GAPDH expression level.

\section{Measurement of intracellular ROS production}

NCSCs were stimulated with BMP2 for 5 min and incubated with $10 \mu \mathrm{M}$ 2',7'-dichlorodihydrofluorescein diacetate (DCF-DA, Molecular Probes) for $10 \mathrm{~min}$. The fluorescence intensity, reflecting intracellular ROS levels, was immediately measured using scanning confocal microscope (LSM 510, Carl Zeiss). The levels of $\mathrm{ROS}$ were expressed in relative fluorescence intensity.

RNA interference for Nox4 knockdown

A lentiviral plasmid vector expressing a short hairpin RNA in
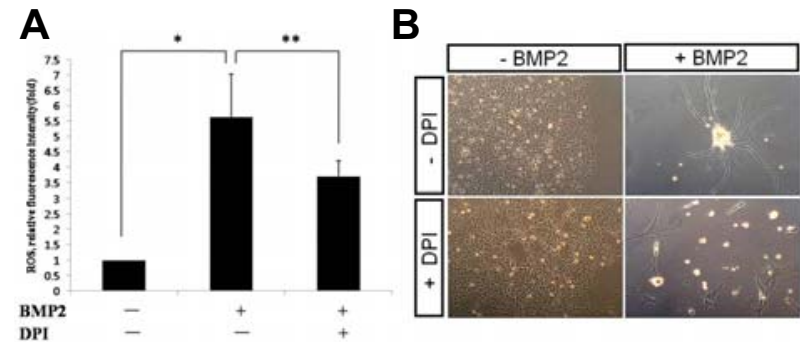

Fig. 2. Neuronal differentiation requires BMP2-induced ROS. (A) NCSCs are treated with BMP2, and ROS generation was measured by green fluorescence from oxidization of DCF-DA into DCFA. Over 5 fold increase is seen ( ${ }^{*} p$ value $<0.005$ by Student's $t$-test). Inclusion of $1 \mu \mathrm{M} \mathrm{DPI}$ reduces fluorescence by approximately $40 \%$ $\left({ }^{* *} p\right.$ value $<0.1$ by Student's $t$-test). (B) In the absence of DPI (upper panels), cells differentiate into large clones in the absence of BMP2 and into mostly neurons in the presence of BMP2. In the presence of $0.1 \mu \mathrm{M}$ DPI (lower panels), BMP2 fails to induce neuronal differentiation.

a microRNA scaffold targeting both rat and mouse Nox4 (pGIPZ-Nox4, oligo ID V2LMM_32043) was obtained from Open Biosystems. Lentiviral vector pGIPZ-Nox4 was cotransfected with viral packaging vectors according to the manufacturer's protocol into HEK-293T cells at $80 \%$ to $90 \%$ cell density using Arrest-In transfection reagent (Open Biosystems). Following $24 \mathrm{~h}$ of incubation, fresh DMEM with $10 \%$ fetal bovine serum was applied to the transfected cells. The medium containing viral particles was harvested after $24 \mathrm{~h}, 48 \mathrm{~h}, 60 \mathrm{~h}$ and $72 \mathrm{~h}$. The supernatant was collected and passed through a $0.45 \mu \mathrm{m}$ filter and concentrated by ultracentrifugation at 35,000 $\mathrm{rpm}$ for $4 \mathrm{~h}$. The pellets were resuspended in serum-free DMEM and flash frozen.

Flow cytometric analysis for apoptosis

NCSCs cells infected either with pGIPZ-Nox4 or control virus were harvested 5 days after infection and examined for apoptosis by staining for Annexin V and PI using ApopNexin FITC Apoptosis Detection Kit for Flow Cytometry (Millipore) or by immunoblotting to examine Caspase 3 activation.

Isolation of Nox4 knockout mouse embryos

Generation and characterization of Nox4 -/- mice have been described (Lee et al., 2013). Genotyping of mouse embryos has been carried out by PCR amplification of genomic DNA derived from embryonic sac. Sequences for the oligonucleotide primers are provided in the supplementary information section (Supplementary Table S2).

Whole mount RNA in situ hybridization

Whole-mount RNA in situ hybridization for mouse embryos was performed as previously described with minor modifications (Birren et al., 1993; Ma et al., 1998). Digoxigenin-labelled probe for NeuroD used in this study has been previously described (Lee et al., 1995). All animal experiments were carried out in accordance with the animal protocol approved by the Animal Care Committee of Ewha Laboratory Animal Genomics Center.

\section{RESULTS}

The protocol for induction of neuronal differentiation of rat NSCSs using BMP2 has been well-established (Shah et al., 1996). 
A
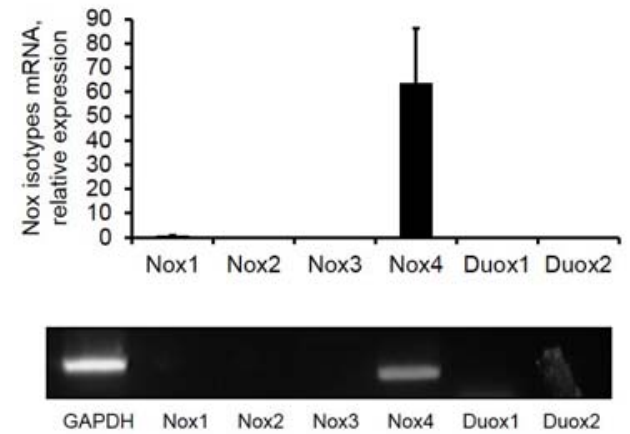

B

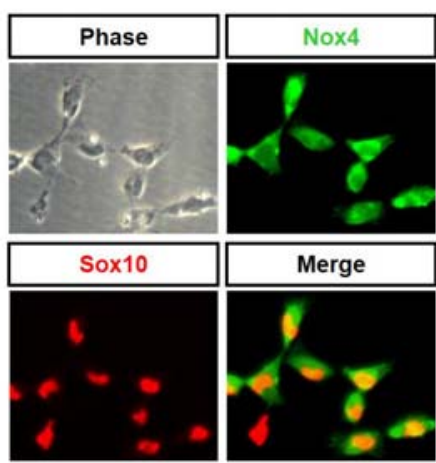

Fig. 3. Nox4 is expressed in NCSCs. (A) RTPCR analysis shows only Nox4 is detected in NCSCs. Results from quantitative RTPCR (upper panel) and gel electrophoresis (lower panel) are shown. (B) Cells double-stained for NCSC-specific nuclear marker Sox10 and Nox4 show clear expression of Nox4 in virtually all NCSCs.

As previously characterized (Kim et al., 2003), while individual NCSCs in control medium expanded into large clones with multiple cell types, BMP2-treated NCSCs differentiated into phase-bright cells with extended axons typical of peripheral neurons generated in vitro (Fig. 1A). The neuronal differentia- tion was also confirmed by staining for Peripherin, a specific marker for fully differentiated neurons (Fig. $1 \mathrm{~B}$ ). We next examined if BMP2 induces generation of ROS using fluorescence from the oxidization of DCF-DA into DCF (Lee et al., 2013). We consistently observed over 5 fold increase in the level of fluorescence of DCFA indicating that BMP2 treatment indeed induced generation of ROS from NCSCs (Fig. 2A). When DPI as a Nox enzyme inhibitor was applied along with BMP2, the level of ROS generation was reduced by about $40 \%$ suggesting that BMP2-mediated ROS generation is through Nox enzyme activity in NCSCs. Importantly, treatment of NCSCs with DPI led to virtual abrogation of BMP2-induced neuronal differentiation over the course of 5 days suggesting that ROS generation is an important part of the differentiation process (Fig. 2B). Of note, NCSCs were exquisitely sensitive to DPI treatment and died en masse requiring the use of DPI level 10 fold lower than the level used in typical cellular experiments (i.e. $0.1 \mu \mathrm{M}$ rather than 1 $\mu \mathrm{M})$. Nevertheless, a large fraction of cells still appeared to have died and surviving cells had the morphology of smooth muscle cells which are known to be generated by BMP2 to a limited extent along with neurons (Shah et al., 1996).

We next sought to determine which of the NAPDH oxidases is expressed and thus likely functional in NCSCs. RTPCR was carried out to this end and only Nox4 was shown to be expressed at a detectable level (Fig. 3A). Nox4 expression was also demonstrated at the protein level using immunocytochemical staining. Specifically, freshly plated NCSCs were doublestained for Sox10, an HMG-box transcription factor and a marker for multipotent NCSCs (Kim et al., 2003; Lee et al., 2008) and Nox4. Virtually all cells were double-positive for the two proteins (Fig. 3B).

To test the function of Nox4 in neuronal differentiation, we sought to down-regulate Nox4 by RNA interference. A lentiviral vector pGIPZ-Nox4 that generates an shRNA embedded in a microRNA context (shRNAmir) and effectively reduces Nox4 RNA level to about $20 \%$ of the wild type level was utilized (Fig. $4 A)$. Interestingly, upon 5 days of culture which is the time required for mature neurons to appear, NCSCs underwent a massive apoptosis (Fig. 4B). Flow cytometric analysis confirmed the microscopic observation and showed a significant increase in Annexin V and/or PI positive cells (Fig. 4C). Further
A

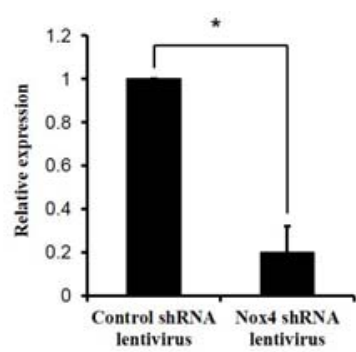

C
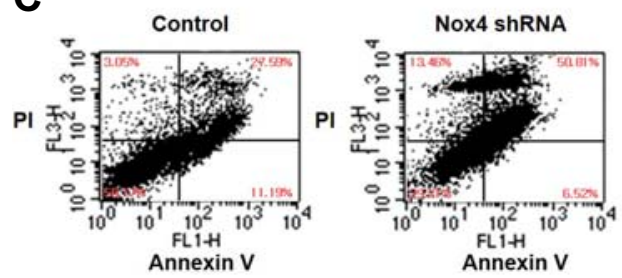

B

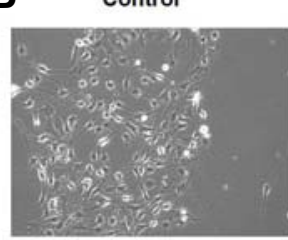

D

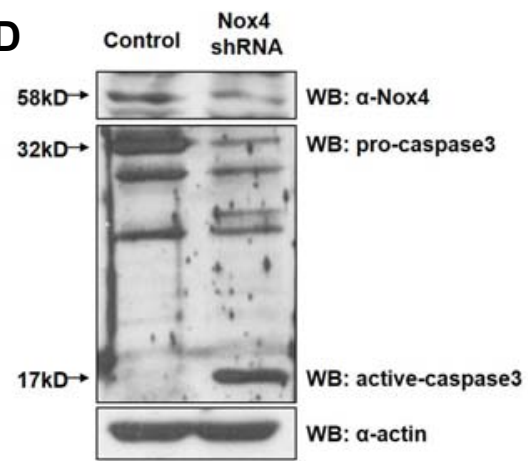

Fig. 4. Nox4 is required for ROS generation and cell survival. (A) pGIPZ-Nox4 virus transduction of NCSCs leads to down-regulation of Nox4 approximately 5 fold compared to control pGIPZ virus transduction ( ${ }^{*} p$ value $<0.001$ by Student's $t$-test). (B) Knock-down of Nox4 induces apoptosis from NCSCs in 5 days. (C) Cells are examined by flow cytometry for apoptosis by $\mathrm{PI}$ and Annexin $V$ staining. pGIPZ-Nox4 virus transduction leads to high level of Annexin $\mathrm{V}$ and PI positive cells consistent with apoptotic induction. (D) Immunoblotting assay shows that pGIPZ-Nox4 virus transduction leads to activation of Caspase 3 which is not seen in control virus transduced cells. 


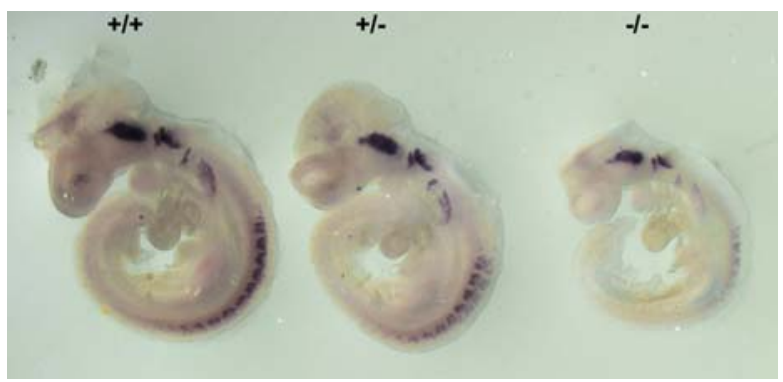

Fig. 5. Expression of peripheral neuronal progenitor marker NeuroD. Littermate embryos from mating of Nox4+/- mice at E9.5 are processed for RNA in situ hybridization with an anti-sense probe specific for NeuroD. Homozygous mutant (-/-) shows retarded growth but proper NeuroD expression in cranial ganglia and dorsal root ganglia commensurate with its size and developmental stage (also see Supplementary Fig. S1).

more, production of active caspase- 3 was significantly increased upon Nox4 down-regulation indicating that NCSCs after Nox4 knockdown underwent apoptotic processes (Fig. 4D).

Given that Nox4 appears to be important for survival of NCSCs in vitro, we next examined the development of neural crestderived peripheral neural structures in Nox4-/- mouse. Nox4-/embryos show generally delayed development and are born at sub-Mendelian ratios. Whole mount RNA in situ hybridization was carried out using early neuronal precursor marker, NeuroD (Lee et al., 1995). In spite of delayed development and smaller size compared to wild type and heterozygote littermates, cranial ganglia and dorsal root ganglia were clearly present in Nox4-/embryo at E9.5 stage (Fig. 5) indicating that neural crest-derived peripheral nervous system is developing at a pace commensurate with the development of the whole embryo. In fact, comparing a better size-matched pair of wild type and Nox4-/- embryos showed normal development of NeuroD-positive cranial and dorsal root ganglia in Nox4-/- embryo (Supplementary Fig. S1). This is also consistent with that surviving Nox4-/- mice are grossly normal with no obvious dysfunction of the peripheral nervous system.

\section{DISCUSSION}

In this report, we examined the involvement of ROS in differentiation of NCSCs. We used a well-established in vitro system using BMP2 as the neuronal differentiation inducer (Shah et al., 1996) and demonstrated that BMP2 indeed induces generation of ROS from NCSCs and that ROS is required for efficient differentiation of NCSCs. That Nox4 is the only detectable NAPDH oxidase at least by RTPCR strongly suggests that the source of ROS is indeed Nox4. However, knockdown of this gene induced apoptotic response from NCSCs making it impossible to dissect detailed function of ROS or Nox4 in neuronal differentiation. Apparently, Nox4 plays other unknown essential roles for the survival of NSCSs at least in vitro. Several lines of evidence indicate that ROS can function in survival of cells through activation of Erk and PI3K/Akt signaling cascades as well as activation of redox-sensitive transcriptional factors including NF- $\mathrm{KB}$, hypoxiainducible factor (HIF)-1a, p53 and AP-1 (Groeger et al., 2009; Kim et al., 2010; Rosc-Schluter et al., 2012; Madenspacher et al., 2013). Specifically, Erk and PI3K/Akt stimulate phosphorylation of pro-apoptotic molecules leading to their inactivation while redox-sensitive transcriptional factors mediate expression of cell survival proteins including SOD and Erk. In addition, several studies have reported that Nox-mediated ROS generation is involved in the cell-to-extracellular matrix (ECM) interaction which often plays critical roles in cell survival (Chiarugi and Giannoni, 2008; Groeger et al., 2009; Paoli et al., 2013). Our results show that silencing of Nox4 in primary NCSCs leads to cell death. We also observed that NCSCs are highly sensitive to DPI treatment necessitating the use of an extremely level to avoid early apoptosis. It is thus highly likely that ROS plays important roles in NCSCs other than regulating neuronal differentiation possibly including controlling interaction with ECM. Of note, NCSCs depend on interaction with a diverse array of ECM for proper development in vivo and survival in vitro (Perris and Perissinotto, 2000; Stemple and Anderson, 1992). Determination of such extra functions of ROS is relegated to future studies.

It is interesting that neural crest-derived peripheral nervous system by and large appears to develop normally in Nox4-/mouse embryos. It is noted that hematopoietic cells including macrophages specifically express Nox2 while muscle cells and connective tissues contain only Nox1. Interestingly, these cells apparently develop more or less normally in mice even when the Nox 2 and Nox1 are deleted by homologous recombination (Gao et al., 2002; Matsuno et al., 2005). It has been thus suggested that other Nox isozymes in surrounding tissues and cells provide ROS during embryonic development. The disparity observed in the survival of NCSCs vivo and in vitro under Nox4-deficient conditions should be an interesting issue for further analysis down the road. Clearly, one possibility is that ROS or ROS-induced extracellular signals are provided by surrounding tissues in vivo during embryogenesis for NCSCs.

Note: Supplementary information is available on the Molecules and Cells website (www.molcells.org).

\section{ACKNOWLEDGMENTS}

This study was supported by grants from the Ministry of Science, ICT and Future Planning via National Research Foundation (2012M3A9B4028785 to YSB and 2012M3A9B4028766 $\mathrm{JK}$ ) and Redoxomics Grant (2012M3A9C5048708). Support also came from the Ministry of Health \& Welfare, Republic of Korea (A120262).

\section{REFERENCES}

Anderson, D.J. (1997). Cellular and molecular biology of neural crest cell lineage determination. Trends Genet. 13, 276-280.

Bae, Y.S., Oh, H., Rhee, S.G., and Yoo, Y.D. (2011). Regulation of reactive oxygen species generation in cell signaling. Mol. Cells 32, 491-509.

Birren, S.J., Lo, L., and Anderson, D.J. (1993). Sympathetic neuroblasts undergo a developmental switch in trophic dependence. Development 119, 597-610.

Buggisch, M., Ateghang, B., Ruhe, C., Strobel, C., Lange, S., Wartenberg, M., and Sauer, H. (2007). Stimulation of ES-cellderived cardiomyogenesis and neonatal cardiac cell proliferation by reactive oxygen species and NADPH oxidase. J. Cell Sci. 120, 885-894.

Chiarugi, P., and Giannoni, E. (2008). Anoikis: a necessary death program for anchorage-dependent cells. Biochem. Pharmacol. 76, 1352-1364.

Gao, X.P., Standiford, T.J., Rahman, A., Newstead, M., Holland, S.M., Dinauer, M.C., Liu, Q.H., and Malik, A.B. (2002). Role of NADPH oxidase in the mechanism of lung neutrophil sequestration and microvessel injury induced by Gram-negative sepsis: studies in p47phox-/- and gp91phox-/- mice. J. Immunol. 168, 3974-3982.

Groeger, G., Quiney, C., and Cotter, T.G. (2009). Hydrogen peroxide as a cell-survival signaling molecule. Antioxid. Redox Signal. 11, 2655-2671. 
Kim, J., Lo, L., Dormand, E., and Anderson, D.J. (2003). SOX10 maintains multipotency and inhibits neuronal differentiation of neural crest stem cells. Neuron 38, 17-31.

Kim, K.S., Choi, H.W., Yoon, H.E., and Kim, I.Y. (2010). Reactive oxygen species generated by NADPH oxidase 2 and 4 are required for chondrogenic differentiation. J. Biol. Chem. 285, 40294-40302.

Le Douarin, N.M. (1980). The ontogeny of the neural crest in avian embryo chimaeras. Nature 286, 663-669.

Lee, J.E., Hollenberg, S.M., Snider, L., Turner, D.L., Lipnick, N., and Weintraub, H. (1995). Conversion of Xenopus ectoderm into neurons by NeuroD, a basic helix-loop-helix protein. Science $268,836-844$.

Lee, K.E., Nam, S., Cho, E.A., Seong, I., Limb, J.K., Lee, S., and $\mathrm{Kim}, \mathrm{J}$. (2008). Identification of direct regulatory targets of the transcription factor Sox10 based on function and conservation. BMC Genomics 9, 408

Lee, J.H., Joo, J.H., Kim, J., Lim, H.J., Kim, S., Curtiss, L., Seong, J.K., Cui, W., Yabe-Nishimura, C., and Bae, Y.S. (2013). Interaction of NADPH oxidase 1 with Toll-like receptor 2 induces migration of smooth muscle cells. Cardiovasc. Res. 99, 483-493.

Lo, L., Dormand, E., Greenwood, A., and Anderson, D.J. (2002). Comparison of the generic neuronal differentiation and neuron subtype specification functions of mammalian achaete-scute and atonal homologs in cultured neural progenitor cells. Development 129, 1553-1567.

Ma, Q., Chen, Z., del Barco Barrantes, I., de la Pompa, J.L., and Anderson, D.J. (1998). neurogenin1 is essential for the determination of neuronal precursors for proximal cranial sensory ganglia. Neuron 20, 469-482.

Madenspacher, J.H., Azzam, K.M., Gowdy, K.M., Malcolm, K.C. Nick, J.A., Dixon, D., Aloor, J.J., Draper, D.W., Guardiola, J.J. Shatz, M., et al. (2013). p53 Integrates host defense and cell fate during bacterial pneumonia. J. Exp. Med. 210, 891-904.

Mandal, C.C., Ganapathy, S., Gorin, Y., Mahadev, K., Block, K., Abboud, H.E., Harris, S.E., Ghosh-Choudhury, G., and GhoshChoudhury, N. (2011). Reactive oxygen species derived from Nox4 mediate BMP2 gene transcription and osteoblast differentiation. Biochem. J. 433, 393-402.
Matsuno, K., Yamada, H., Iwata, K., Jin, D., Katsuyama, M. Matsuki, M., Takai, S., Yamanishi, K., Miyazaki, M., Matsubara, $\mathrm{H}$., et al. (2005). Nox1 is involved in angiotensin II-mediated hypertension: a study in Nox1-deficient mice. Circulation 112, 2677-2685.

Milosevic, N., Bekhite, M.M., Sharifpanah, F., Ruhe, C., Wartenberg, M., and Sauer, H. (2010). Redox stimulation of cardiomyogenesis versus inhibition of vasculogenesis upon treatment of mouse embryonic stem cells with thalidomide. Antioxid. Redox. Signal. 13, 1813-1827.

Morrison, S.J., White, P.M., Zock, C., and Anderson, D.J. (1999). Prospective identification, isolation by flow cytometry, and in vivo self-renewal of multipotent mammalian neural crest stem cells. Cell 96, 737-749.

Nadworny, A.S., Guruju, M.R., Poor, D., Doran, R.M., Sharma, R.V. Kotlikoff, M.l., and Davisson, R.L. (2013). Nox2 and Nox4 influence neonatal c-kit(+) cardiac precursor cell status and differentiation. Am. J. Physiol. Heart Circ. Physiol. 305, H829-842.

Paoli, P., Giannoni, E., and Chiarugi, P. (2013). Anoikis molecular pathways and its role in cancer progression. Biochim. Biophys. Acta 1833, 3481-3498.

Perris, R., and Perissinotto, D. (2000). Role of the extracellular matrix during neural crest cell migration. Mech. Dev. 95, 3-21.

Rosc-Schluter, B.I., Hauselmann, S.P., Lorenz, V., Mochizuki, M. Facciotti, F., Pfister, O., and Kuster, G.M. (2012). NOX2-derived reactive oxygen species are crucial for CD29-induced prosurvival signalling in cardiomyocytes. Cardiovasc. Res. 93, 454462.

Shah, N.M., Groves, A.K., and Anderson, D.J. (1996). Alternative neural crest cell fates are instructively promoted by TGFbeta superfamily members. Cell $85,331-343$

Simone, S., Cosola, C., Loverre, A., Cariello, M., Sallustio, F. Rascio, F., Gesualdo, L., Schena, F.P., Grandaliano, G., and Pertosa, G. (2012). BMP-2 induces a profibrotic phenotype in adult renal progenitor cells through Nox4 activation. Am. J. Physiol. Renal. Physiol. 303, F23-34.

Stemple, D.L., and Anderson, D.J. (1992). Isolation of a stem cell for neurons and glia from the mammalian neural crest. Cell 71 973-985. 